\title{
Analysis of Impact of Channel Error Rate on Average PSNR in Multimedia Traffic
}

\author{
Kirandeep Singh ${ }^{1}$, Er. Harshit ${ }^{2}$, Dr. KVP Singh ${ }^{3}$, Dr. Nipun Sharma ${ }^{4}$ \\ ${ }^{1}$ Research Scholar, Pursuing M.Tech (ECE), DIET, Kharar, Distt - Mohali (Punjab) - 140103 INDIA \\ ${ }^{2}$ A.P. \& HoD (ECE), DIET, Kharar, Distt - Mohali (Punjab) - 140103 INDIA \\ 3Director Principal, DIET, Kharar Distt - Mohali (Punjab) - 140103 INDIA \\ ${ }^{4}$ AP, Quest Group of Institutions, Mohali, (Punjab) - INDIA
}

Abstract: The performance of the multimedia traffic in Ad-Hoc networks is highly impacted with the Signal to Noise Ratio. The Average PSNR (Peak Signal to Noise Ratio) is an important parameter for the evaluation of multimedia traffic in Ad-Hoc Networks. With the increase of bandwidth of the channels, it becomes necessary to take care of other network parameters like PSNR and ASNR(Average Signal to Noise Ratio). Enhanced bandwidth with higher channel error rates demand a careful analysis of signal to noise ratio for optimum performance. In this paper, we have evaluated the effect of channel error rate on Average PSNR for the MPEG4 traffic in Ad-hoc Networks.

Keywords: MANETS, Evalvid, MPEG-4, Fragmentation, PSNR

\section{Introduction}

Mobile Ad-hoc Networks are typical examples of infrastructure-less networks and are established on the go. A lot of work on the improvement of routing of data in MANETs for different classes has been done; latest being the multimedia traffic class. With more and more content on the network becoming audio-visual, it essentially becomes important to study the analysis of multimedia in Mobile Ad-hoc Networks.

This paper discusses the implementation of fragmentation on the multimedia traffic class, i.e. MPEG-4 in Mobile Ad-hoc Networks. The NS-2 is the most popular simulator for the simulation of Mobile Ad-hoc Networks. In order to evaluate multimedia traffic in NS-2, a framework EVALVID is integrated to NS-2. Evalvid is a tool-set which is usually evaluating the quality of video. This framework measures the various QOS parameters like packet loss, delay etc. It also predicts the evaluation of video quality which is received at the receiver side based on the calculation of PSNR [1].

\section{MPEG-4}

MPEG-4 (Motion Pictures Expert Group-4) standard was introduced in late 1998. This standard enables the compression of audio-visual data at better quality. This standard supports specific functions in the form of efficiency, interactivity etc. [2].

The MPEG-4 standard defines three types of video frames for the compressed video stream, including I (Intra-coded) frame, P (Predictive-coded) frame, and B (Bi-directionally predictive-coded) frame. The MPEG I frame is encoded independently and decoded by itself. Thus, the I frame is just a frame coded as a still image, without any relationship to any previous or successive frames. The $\mathrm{P}$ frame is encoded using prediction from the preceding I or $\mathrm{P}$ frame in the video sequence. Thus the $\mathrm{P}$ frame requires the information of the most recent $\mathrm{I}$ frame or P frame for encoding and decoding. The B frame is encoded using predictions from the preceding and succeeding I or P frames. According to the coding relation, in MPEG-4 video stream the most important video type is the I frame, with the $\mathrm{P}$ frame being more important than B frame.

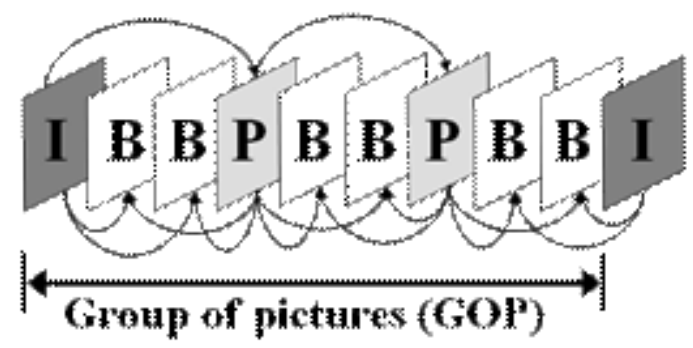

Figure 1: Prediction encoding of MPEG-4, GOP $(\mathrm{N}=9, \mathrm{M}=3)$

The video sequence can be decomposed into smaller units, GOP (Group Of Picture), similar to a deterministic periodic sequence of frames (as shown in Figure 1). A GOP pattern is characterized by two parameters, G (N, 
$\mathrm{M})$ : $\mathrm{N}$ is the I-to-I frame distance and $\mathrm{M}$ is the I-to-P frame distance. For example, $\mathrm{G}(9,3)$ means that the GOP includes one I frame, two P frames, and six B frames. Similarly, the second I frame in the figure marks the beginning of the next GOP. The arrows indicate that the B frames and P frames decoded are dependent on the preceding or succeeding I or P frames.

\section{PSNR (Peak Signal Noise Ratio)}

PSNR is one of the most widespread objective metrics to assess the application-level QoS of video transmissions. The following equation shows the definition of the PSNR between the luminance component Y of source image $\mathrm{S}$ and destination image $\mathrm{D}: \operatorname{PSNR}(\mathrm{n}) \mathrm{dB}=20$

$$
\log 10\left(\frac{V_{p e a k}}{\sqrt{\frac{1}{N_{c o l} N_{r o w}} \sum_{i=0}^{N_{c o l} N_{\text {row }}} \sum_{j=0}\left[Y_{S}(n, i, j)-Y_{D}(n, i, j)\right]^{2}}}\right),
$$

where $\mathrm{V}_{\text {peak }}=2 \mathrm{k}-1$ and $\mathrm{k}=$ number of bits per pixel (luminance component). PSNR measures the error between a reconstructed image and the original one. Prior to transmission, one may then compute a reference PSNR value sequence on the reconstruction of the encoded video as compared to the original raw video. After transmission, the PSNR is computed at the receiver for the reconstructed video of the possibly corrupted video sequence received. The individual PSNR values at the source or receiver do not mean much, but the difference between the quality of the encoded video at the source and the received one can be used as an objective QoS metric to assess the transmission impact on video quality at the application level.

\section{Methodology}

The simulator environment is based on Cygwin installed on Windows-XP operating system. The simulation consists of two nodes node -1 and node -2 which are exchanging a multimedia file (i.e. MPEG File). Node -1 is source and node- 2 is sink. The traffic is routed using AODV Protocol. The total size of the MPEG file is sent approximately $50 \mathrm{Mb}$. The simulation setup is designed to analyze the performance metric-"fraction of decodable frames".

The data transfer takes place from node-1(source) to node-2(sink) in packets. The number of packets depends upon the frame size. If the frame size is small the number of packets is large i.e. fragmentation is more, if the frame size is large the number of packets will be small i.e. the fragmentation is less. We have tested the MPEG-4 Traffic for the following "maximum frame size" parameter:

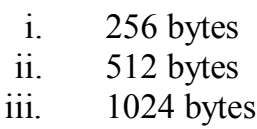

The following channel error rates $[0.1,0.2,0.3,0.4,0.5,0.6,0.7$, and 0.8$]$ are being considered for the experiment.

The Simulation results show fragment size deteriorates the PSNR at moderate channel error rates i.e $(0.2,0.4,0.6)$. When the channel error rate is very low i.e. (0.1) lower fragment size gives the best ASNR. When the channel error rate is kept very high i.e. (0.8) ASNR is independent of fragment size.

The exact simulation results are presented in a tabular form below.

\begin{tabular}{|r|r|r|r|r|r|r|r|r|}
\hline \multicolumn{7}{|l|}{ ASNR } & \multicolumn{7}{|c|}{ Channel Error Rate } \\
\hline $\begin{array}{l}\text { Fragment } \\
\text { Size }\end{array}$ & 0.1 & 0.2 & 0.3 & 0.4 & 0.5 & 0.6 & 0.7 & 0.8 \\
\hline 256 & 0 & 97.00991 & 126.7748 & 85.14923 & 59.01955 & 49.23265 & 45.45526 & 31.03018 \\
\hline 512 & 0 & 59.87102 & 104.8033 & 71.2341 & 49.63259 & 57.0431 & 43.55012 & 33.88526 \\
\hline 1024 & 0 & 21.45791 & 59.41914 & 47.1154 & 45.35974 & 43.2128 & 36.16 .21 & 30.46889 \\
\hline
\end{tabular}

Table 1. ASNR for Different Channel Error rates at different Fragment Sizes 
The Simulation results in the form of graph are as follows.

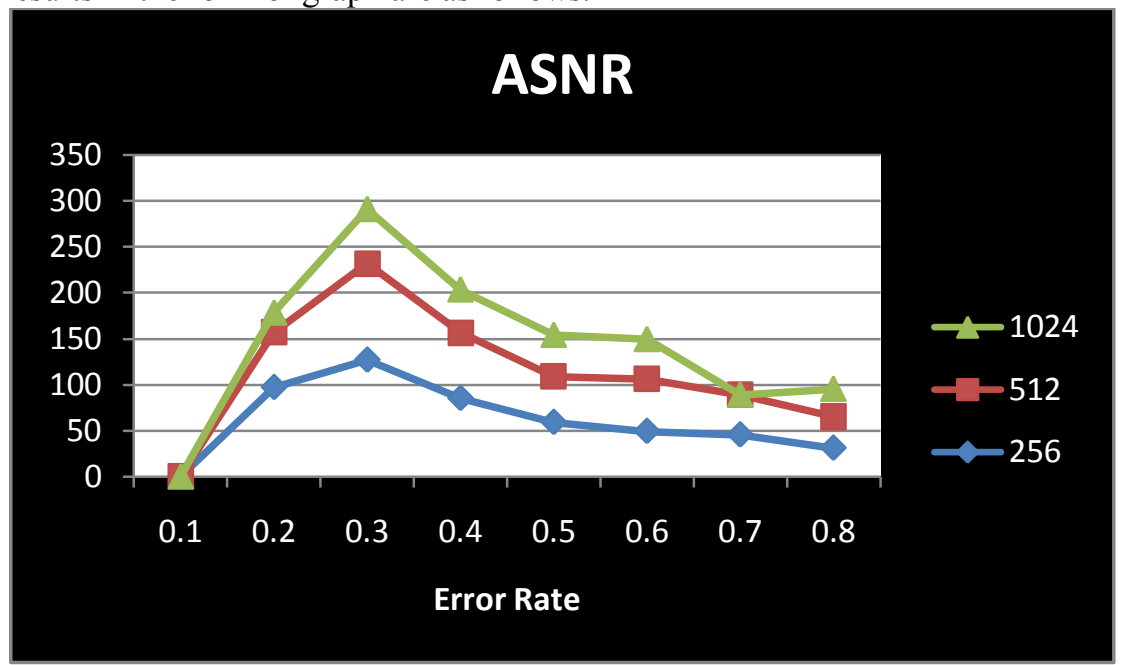

Fig 1. Line View of Error Rate versus the ASNR at different Fragment Sizes

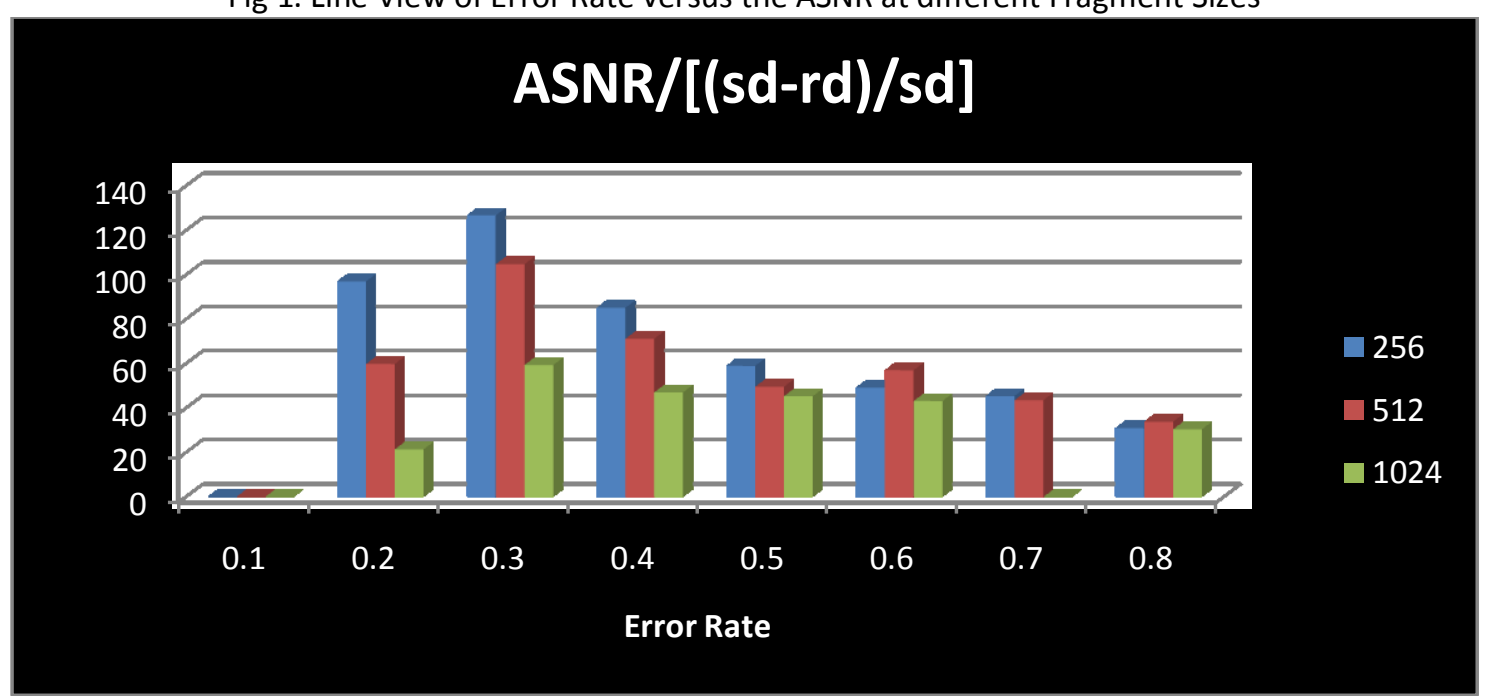

Fig 2. 3D view of Error Rate versus the ASNR at different Fragment Sizes

\section{Conclusion and Future Scope}

The objective of the work is to understand and analyze the effect of fragmentation on the multimedia traffic over UDP to monitor ASNR for different channel error rate. The effect of fragmentation on PSNR is sporadic and the optimization requires a careful calibration. The study can be extended with the TCP traffic also under various routing protocols.

\section{References:}

[1] Thomas D. Dyer Rajendra V. Boppana Computer Science Department, the Univ. of Texas at San Antonio, San Antonio, TX 78249 tdyer@cs.utsa.edu boppana@cs.utsa.edu “On Routing Web and Multimedia Traffic in Mobile Ad Hoc Networks” Proceedings of the 36th Hawaii International Conference on System Sciences (HICSS’03) 0-7695-1874-5/03@ 2002 IEEE.

[2] MPEG-4 Natural Video Coding - An overview Touradj Ebrahimi* and Caspar Horne** * Signal Processing Laboratory **Mediamatics Inc. Swiss Federal Institute of Technology - EPFL 48430 Lakeview Blvd 1015 Lausanne, Switzerland.

[3] Klaue, J., Rathke, B. \& Wolisz, A. (2003), "A Framework for Video Transmission and Quality Evaluation- EvalVid", Proceedings of the 13th International Conference on Modelling Techniques and Tools forComputing Performance Evaluation, $255-272$. http://www.tkn.tu-berlin.de/research/evalvid.

[4] Lo, A., Heijenk, G. \& Niemegeers, I. (2005), "Performance Evaluation of MPEG-4 Video Streaming over UMTS Networks using an Integrated Tool Environment", Proceedings of 2005 International Symposium on Performance Evaluation of Computer and Telecommunication Systems, 676-682.

[5] C. H. Ke, C. K. Shieh, W. S. Hwang, A. Ziviani, “An Evaluation Framework for More Realistic Simulations of MPEG Video Transmission", Journal of Information Science and Engineering, vol. 24, no. 2, pp.425-440, March 2008 (SCI)

[6] Sheeraz Ahmed'll, Muhammad Bilailr], Umer Farooq'l], Fazl-e-Hadi,N-W.F.P University of Engineering and Technology, Peshawar, Pakistan "Performance Analysis of various routing Strategies in Mobile Ad hoc Network using QualNet simulator" 14244-1494-6/07/ C 2007 IEEE.

[7] Network Simulator 2 http://www.isi.edu/nsnam/ns/ 
[8] Md. Anwar Hossain, A.N.M. Bazlur Rashid, Department of Information and Communication Engineering, Pabna Science and TechnologyUniversity, Pabna, Bangladesh "Performance Evaluation of MPEG-4 Video Transmission over IP-Networks: BestEffort and Quality-of-Service" Computer Engineering and Intelligent Systems ISSN 2222-1719 (Paper) ISSN 2222-2863 (Online) Vol 3, No.3, 2012 . 